Article

\title{
Multistage Chemical Recycling of Polyurethanes and Dicarbamates: A Glycolysis-Hydrolysis Demonstration
}

\author{
Pegah Zahedifar, Lukasz Pazdur*(D, Christophe M. L. Vande Velde ${ }^{(D)}$ and Pieter Billen \\ Faculty of Applied Engineering, iPRACS, University of Antwerp, 2020 Antwerp, Belgium; \\ zahedifar.pegah@gmail.com (P.Z.); christophe.vandevelde@uantwerpen.be (C.M.L.V.V.); \\ pieter.billen@uantwerpen.be (P.B.) \\ * Correspondence: lukasz.pazdur@uantwerpen.be; Tel.: +32-3-265-88-65
}

check for updates

Citation: Zahedifar, P.; Pazdur, L.; Vande Velde, C.M.L.; Billen, P. Multistage Chemical Recycling of Polyurethanes and Dicarbamates: A Glycolysis-Hydrolysis Demonstration. Sustainability 2021, 13, 3583. https:// doi.org/10.3390/su13063583

Academic Editor: Edoardo Bocci

Received: 27 February 2021

Accepted: 19 March 2021

Published: 23 March 2021

Publisher's Note: MDPI stays neutral with regard to jurisdictional claims in published maps and institutional affiliations.

Copyright: (c) 2021 by the authors. Licensee MDPI, Basel, Switzerland. This article is an open access article distributed under the terms and conditions of the Creative Commons Attribution (CC BY) license (https:// creativecommons.org/licenses/by/ $4.0 /)$.
Abstract: The use of polyurethanes and, therefore, the quantity of its scrap are increasing. Considering the thermoset characteristic of most polyurethanes, the most circular recycling method is by means of chemical depolymerization, for which glycolysis is finding its way into the industry. The main goal of polyurethane glycolysis is to recover the polyols used, but only limited attempts were made toward recovering the aromatic dicarbamate residues and derivates from the used isocyanates. By the split-phase glycolysis method, the recovered polyols form a top-layer phase and the bottom layer contain transreacted carbamates, excess glycol, amines, urea, and other side products. The hydrolysis of carbamates results in amines and $\mathrm{CO}_{2}$ as the main products. Consequently, the carbamates in the bottom layer of polyurethane split-phase glycolysis can also be hydrolyzed in a separate process, generating amines, which can serve as feedstock for isocyanate production to complete the polyurethane material cycle. In this paper, the full recycling of polyurethanes is reviewed and experimentally studied. As a matter of demonstration, combined glycolysis and hydrolysis led to an amine production yield of about $30 \%$ for model systems. With this result, we show the high potential for further research by future optimization of reaction conditions and catalysis.

Keywords: recycling; chemical recycling; polyurethanes; carbamates; glycolysis; hydrolysis

\section{Introduction}

Polyurethanes (PUs) are among the most used polymers with an estimated global annual production of around 28 million metric tons in 2020 [1] and are the fifth most used polymer in Europe with a demand of approximately 4 million tons in 2018 and 2017 out of the overall polymer demand of 51.2 million tons [2]. Polyurethanes are formed by a condensation polymerization reaction between polyisocyanates and polyols [3]. The reaction between an alcohol with $-\mathrm{OH}$ functional group and an isocyanate with -NCO functional group leads to a urethane (derived from urea-ethane, which is in fact a carbamate bond), as discovered by Bayer and coworkers in 1937 [4]. Reaction (a) on Scheme 1 shows carbamate (or urethane bond) formation from isocyanate reacting with an alcohol.

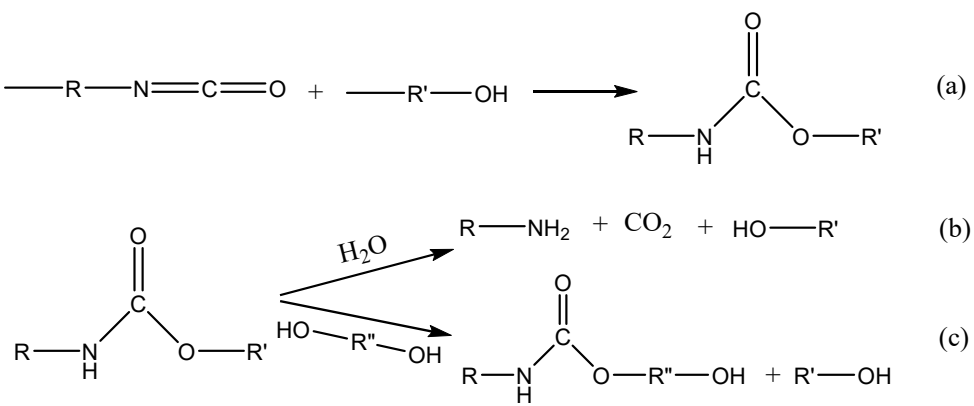

Scheme 1. Synthesis (a) and decomposition of urethane bond; (b) hydrolysis; (c) glycolysis. 
The properties of polyurethanes can vary greatly based on the type of polyols and isocyanates used, their formulation, and also the additives, such as blowing agents and dyes, used during the production process. The commonly used polyols in the production of polyurethanes are polyether polyols either based on polypropylene, polyethylene oxide or tetrahydrofuran, aliphatic polyester polyols, aromatic polyester polyols, polycarbonate polyols, acrylic polyols, and polybutadiene polyols [5]. The isocyanates mostly employed for polyurethanes can be categorized into two groups of aromatics; toluene diisocyanate (TDI) and methylene diphenyl diisocyanate (MDI). In a few applications, aliphatic isocyanates such as isophorone diisocyanate (IPDI) and hexamethylene diisocyanate (HDI) are used. The aromatic types are more reactive and used in many common applications such as flexible and rigid foams [5]. In the synthesis of polyurethanes, in manufacturing processes or in-situ, a number of side reactions typically occur leading to the formation of allophanate, uretonimine, urea, biuret, amine, and carbodiimide moieties [6]. These side reactions are the main reason of the complexity of polyurethane formulations and also the crosslinking of the bonds for thermoset polyurethanes.

Due to a wide range of achievable properties, numerous varieties of polymer formulations exist, each with different applications, among which rigid foams, flexible foams, binders, coatings, sealants, and elastomers are some of the examples [5]. The high demand in polyurethane production leads to a large quantity of industrial and post-consumer wastes to be treated. Landfilling of plastics, in general, occupies valuable space, and it takes a long time for plastics to be degraded [7,8]. On the other hand, landfilled polyurethanes could release toxic chemicals such as amines into the soil [9]. Additionally, although there are many studies regarding the high energy recovery from polyurethanes by incineration, it leads to a loss of the intrinsic energy of the polymer, failing to fulfil the existing material demand with other-than-virgin resources $[10,11]$. Consequently, plastic recycling is a promising method for waste management, overcoming the aforementioned problems. In the case of polyurethanes, recycling technologies are classified into two types: mechanical and chemical recycling [12].

Although thermoplastic polyurethanes are used in some fields of industry, most of the synthesized polyurethanes are thermosets. The crosslinked bonds of thermoset polymers hinder the re-melting and re-shaping of the polymers, and so prevalent mechanical recycling methods, commonly used for thermoplastics such as polyethylene or polystyrene, are not applicable. Mechanical recycling of polyurethanes therefore is confined to methods such as regrinding, re-bonding, adhesive pressing, compression molding, and injection molding of the polyurethane particles along with a molten polymer or additional isocyanate monomer resin [13-15]. The advantage of these methods is their low cost and simplicity, but the applicability is limited [10]. Indeed, the obtained products are not comparable to virgin foams, and therefore, these materials are predominantly directed to niche applications with-for the time being - a modest market size. Examples of such niche applications are acoustic insulation or circular mattresses. Alternatively, they are used in lower-value applications such as underlays in livestock stables.

The history of the chemical recycling of polyurethanes is nearly as old as this polymer itself $[16,17]$. Chemical recycling of polymers is generally defined as a process to recover the initial or alternative monomers or building blocks, which in the case of polyurethanes are the polyols and isocyanates or amines [18]. When amines are obtained, they can be converted back into isocyanates by reaction with phosgene. By this method, raw materials are obtained, which can be re-used in the production of polyurethanes or other heteropolymers. The chemical recycling methods studied for polyurethanes include phosphorolysis, aminolysis, hydrolysis, and glycolysis, among which the latter two have gained the most attention.

Hydrolysis is the (catalyzed) reaction of water with polyurethane at high temperatures and pressures to form polyols, amines, and $\mathrm{CO}_{2}$ gas, which can be seen from reaction (b) on Scheme 1. In fact, during this reaction, carbamic acid is formed first, which is thermally unstable and subsequently decomposes into amine and $\mathrm{CO}_{2}$. Several attempts 
have been made to optimize the hydrolysis conditions of polyurethanes and gain the highest efficiency [19-21]. Thus far, to our knowledge, hydrolysis-based recycling methods were limited to pilot plants and not further industrially implemented, mainly due to the high temperature and pressure needed [22].

Glycols are bifunctional alcohols with relatively high boiling points, exceeding $197^{\circ} \mathrm{C}$ (for ethylene glycol), which can be used as decomposition agents for polyurethanes. Glycolysis is the most important chemical recycling method for polyurethanes to date, to be developed at industrial scale. The method consists of a transcarbamation reaction between the original polyol connected to the carbamate group and the hydroxyl group of glycol as shown in reaction (c) on Scheme 1. This transcarbamation reaction is comparable to transesterifications. Decades of research on the glycolysis of polyurethanes, either to assess the result of this reaction or for the optimization of its conditions, have resulted in a variety of potential setups. To date, the polyols obtained from split-phase glycolysis show better properties and have higher purity compared to the polyols recovered from single-phase glycolysis. Split-phase glycolysis is mainly obtained by using a stoichiometric excess of glycols for the recycling of polyurethanes containing high-molecular-weight polyols. The solubility of the polyols in the glycol plays an important role in the separation of two phases. By this method, the upper phase consists mostly of the recovered polyols, and the bottom phase is the excess glycol and other products of the reaction [23].

Different glycolysis agents have been studied for polyurethane foam recycling. The glycolysis of polyurethane foam in split phase with different reagents including ethylene glycol, 1,2-propylene glycol, triethylene glycol, polyethylene glycol, diethanolamine, and their mixtures without catalyst showed that using ethylene glycol leads to lower kinematic viscosity of the recovered polyol fraction, which is more desirable for further utilization [24]. In other work, the efficiency of ethylene glycol and that of diethylene glycol as decomposition agents for the glycolysis of refrigerator (rigid) foams were compared, showing that ethylene glycol is a better agent than diethylene glycol, as the reaction is faster at lower temperatures, owing to its simpler spatial structure, which avoids steric hindrance [25]. The choice of catalyst is also an important factor during the glycolysis of polyurethane foams. The most important catalysts for polyurethane glycolysis are sodium hydroxide, potassium hydroxide, sodium acetate, potassium acetate, stannous octanoate, and amines [25-27]. The choice of reaction temperature depends on multiple reaction parameters, such as the choice of catalyst and glycolysis agent. The reaction temperature can be lowered to around $170{ }^{\circ} \mathrm{C}$ to avoid unwanted thermal decomposition reactions of the polyurethane during glycolysis [24] or can be as high as around the boiling point of the glycolysis agent. It is shown that increasing the temperature would lead to decomposition of the reactants and the production of unwanted amines, which has an adverse effect on the purity of recovered polyols [28-32].

Beside the recovered polyols, the split-phase glycolysis results in a second phase containing the excess glycols, carbamates, amines, low-molecular-weight polyols, and other chemicals produced during the decomposition reaction. Due to the toxic effect of amines on the environment and (aquatic) organisms, this phase of glycolysis should not be disposed [33-35]. Most studies do not further discuss this phase, assuming it would be incinerated. In a few studies, this phase, constituting the bottom of the split-phase glycolysis, has been tried for use in other applications in order to avoid disposal. For example, in the work of Simon et al. [36], the glycolysis bottom phase was used as the initiator in the anionic polymerization of polyether polyols synthesis. The excess glycol was distilled from the bottom phase, and the residue, which mostly contains aromatic amines and transreacted carbamates, was used for the polymerization. This residue possessed active hydrogens, which made it suitable to replace the polyether polyol initiator [36]. Additionally, the transreacted carbamates may have many applications in industry if purified, although their separation is not straightforward. Indeed, carbamates could be used as herbicides, and therefore, their stability in the aquatic environment is important. The kinetics and mechanism of their hydrolysis reaction has been assessed in several 
studies $[37,38]$. Furthermore, carbamate hydrolysis is used as a technique to improve the separation and detection of this moiety in capillary electrophoresis [39]. In a similar way, for the prediction of thermal breakthrough in an attempt at geothermal reservoir management, carbamates were used as thermo-sensitive tracers [40].

In this regard, the hydrolysis kinetics of primary and secondary carbamates were investigated. There are two mechanisms for carbamate hydrolysis, as shown in Scheme 2. Reaction (a), via an E1cb mechanism, is related to primary carbamates, and reaction (b), via the $\mathrm{B}_{\mathrm{AC}} 2$ mechanism, is related to secondary carbamates, in which there is an alkyl/aryl group at $R_{2}$ instead of $H$. The temperature and structure of carbamates significantly influence the kinetics of hydrolysis, whereas the $\mathrm{pH}$ has a minor effect [40].

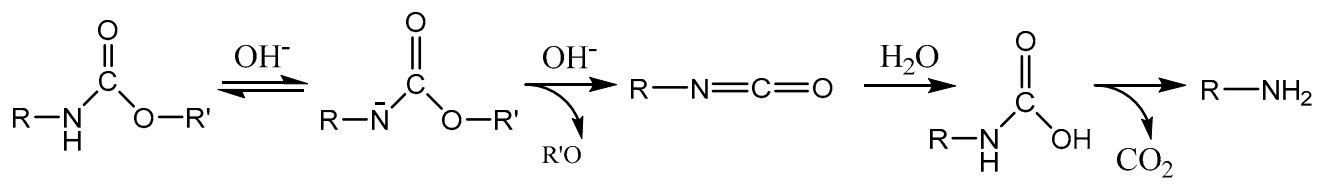

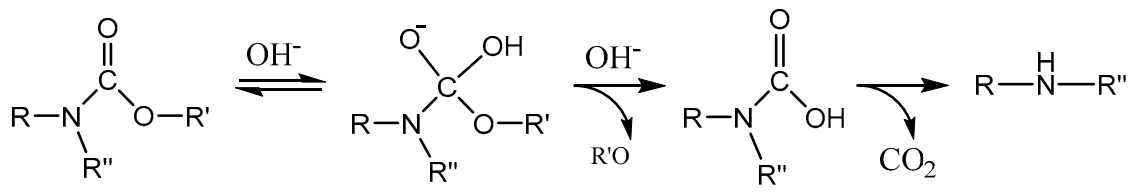

Scheme 2. Carbamate base-catalyzed hydrolysis mechanisms, including (a) E1cb (primary carbamates) and (b) $\mathrm{B}_{\mathrm{AC}} 2$ (secondary carbamates).

The hydrolysis of carbamates could and should also be employed as a recycling strategy for the aforementioned glycolysis residue. In this way, the carbamate residue can be decomposed to precious chemicals such as amines, which subsequently are feedstock in the production process of isocyanates. This would complete the polyurethane material cycle.

On the other hand, the hydroglycolysis of polyurethane known as soft glycolysis has gained attention due to the milder conditions of this reaction. It is in fact an integrated recycling operation combining hydrolysis and glycolysis in a single step. This reaction is done by using water and alcohol as decomposition agents at $100^{\circ} \mathrm{C}$ for $24 \mathrm{~h}$ [41]. Moreover, it is claimed that polyols reclaimed in a glycolysis reaction with the addition of a small amount of water have higher purity [42,43]. Additionally, the hydroglycolysis has been explored at atmospheric pressure in microwave reactors [44].

In this paper, the multistage chemical recycling of polyurethane and its dicarbamates is demonstrated to assess the potential for complete recovery of the monomers. The present work aims at advancing the state of the art with insights into how PU can be fully recycled, instead of only focusing on polyols. A conventional glycolysis is first executed to recover the polyol on the one hand and to obtain smaller carbamates on the other hand. Then, these new carbamates are separated and used for a further hydrolysis step to recover amines, which can be re-processed into isocyanates. For the sake of clarity, avoiding mixtures from industrial polyurethane waste that are too complex to characterize, model polyurethanes and dicarbamates were used in this study. In order to obtain insights about reactivity, dicarbamates of either diols with monoisocyanates or alcohols with diisocyanates were investigated, in addition to synthesized polyurethane.

\section{Materials and Methods}

All the materials and reagents used for synthesis, glycolysis, and hydrolysis in this research are analytical grade and purchased from Sigma Aldrich (Overijse, Belgium).

\subsection{Dicarbamate Synthesis}

Hexane-1,6-diyl bis(phenylcarbamate) (1) (reaction (a) on Scheme 3) was synthesized as follows: $3 \mathrm{~g}$ (1 eq.) of 1,6-hexanediol, $5.1 \mathrm{~g}$ (2 eq.) of phenyl isocyanate and $0.5 \mathrm{~g}$ 
(0.001 eq.) of tin 2-ethylhexanoate as catalyst were added to a three-neck flask under argon atmosphere, and the mixture was heated to $100{ }^{\circ} \mathrm{C}$ for two hours. Dihexyl 1,4phenylenedicarbamate (2) (reaction (b) on Scheme 3) was synthesized under the same conditions using $5.0 \mathrm{~g}$ ( 2 eq.) of 1-hexanol and $3.0 \mathrm{~g}$ (1 eq.) of phenylene diisocyanate in the presence of $0.5 \mathrm{~g}$ ( 0.001 eq.) of tin 2-ethylhexanoate as catalyst for two hours. Then, the reaction mixture was cooled to room temperature, and $2 \mathrm{~mL}$ of water was added to neutralize the catalyst and non-reacted isocyanate. The mixture was transferred to a separation funnel and extracted three times with MTBE $(3 \times 10 \mathrm{~mL})$. The organic fractions were combined, dried over $\mathrm{MgSO}_{4}$, filtered, and the solvent was removed on the rotavapor.

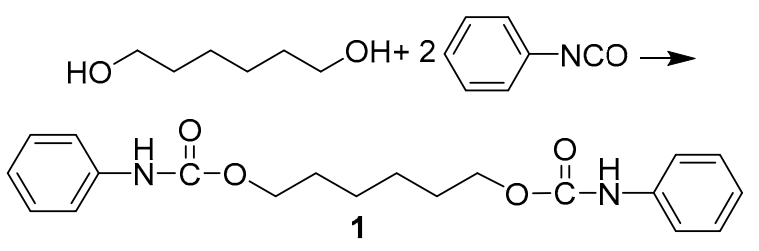

hexane-1,6-diyl bis(phenylcarbamate)

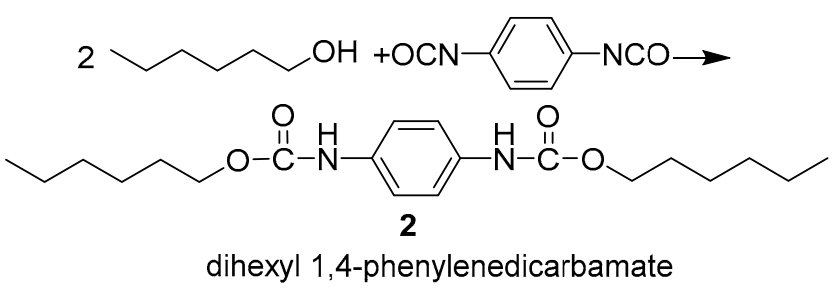

(b)

Scheme 3. Synthesis of dicarbamates.

\subsection{Model Polyurethane Synthesis}

In order to avoid the influence of the complications that exist in industrial polyurethane structures on the procedure and results, a model polyurethane (3) (Scheme 4), based on phenylene diisocyanate and 1,6-hexanediol, was synthesized and subsequently used for recycling in this research. DMF was used as the solvent medium for the synthesis [45]. To synthesize polyurethane (3) (Scheme 4) $20 \mathrm{~mL}$ of DMF containing $2.9 \mathrm{~g}$ (1 eq.) of 1,6hexanediol, $20 \mathrm{~mL}$ solution of DMF containing $3.9 \mathrm{~g}$ (1 eq.) of phenylene diisocyanate, and $0.5 \mathrm{~g}$ (0.001 eq.) of tin 2-ethylhexanoate as the catalyst were added to a three-neck flask under argon atmosphere, and the mixture was heated to $120^{\circ} \mathrm{C}$ for two hours. Then, the reaction mixture was cooled to room temperature, and $2 \mathrm{~mL}$ of water was added to neutralize the catalyst and non-reacted isocyanate. The precipitate (product) was filtered, washed with water, and dried overnight.

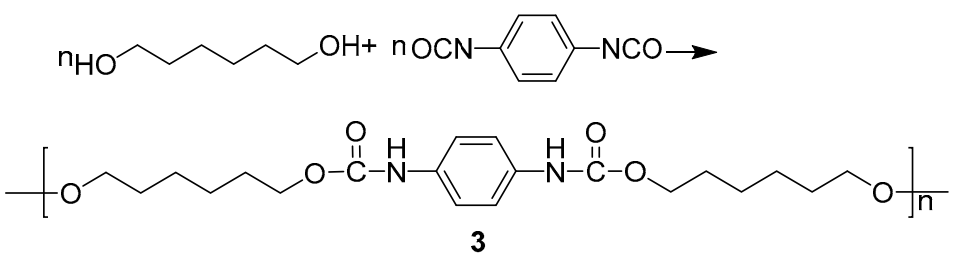

Scheme 4. Synthesis of model polyurethane.

After the synthesis of dicarbamates ( $\mathbf{1}$ and $\mathbf{2}$ ) and polyurethane (3), they were used for multistage recycling. The overview of this recycling is shown in Scheme 5 . It can be seen that at first glycolysis was conducted (1a, 2a, 3a), which would lead to polyol recovery and for the next step, hydrolysis was executed on the carbamate product of glycolysis $(1 b, 2 b, 3 b)$. As a result, amines were formed. These steps are explained in detail in the next sections. 


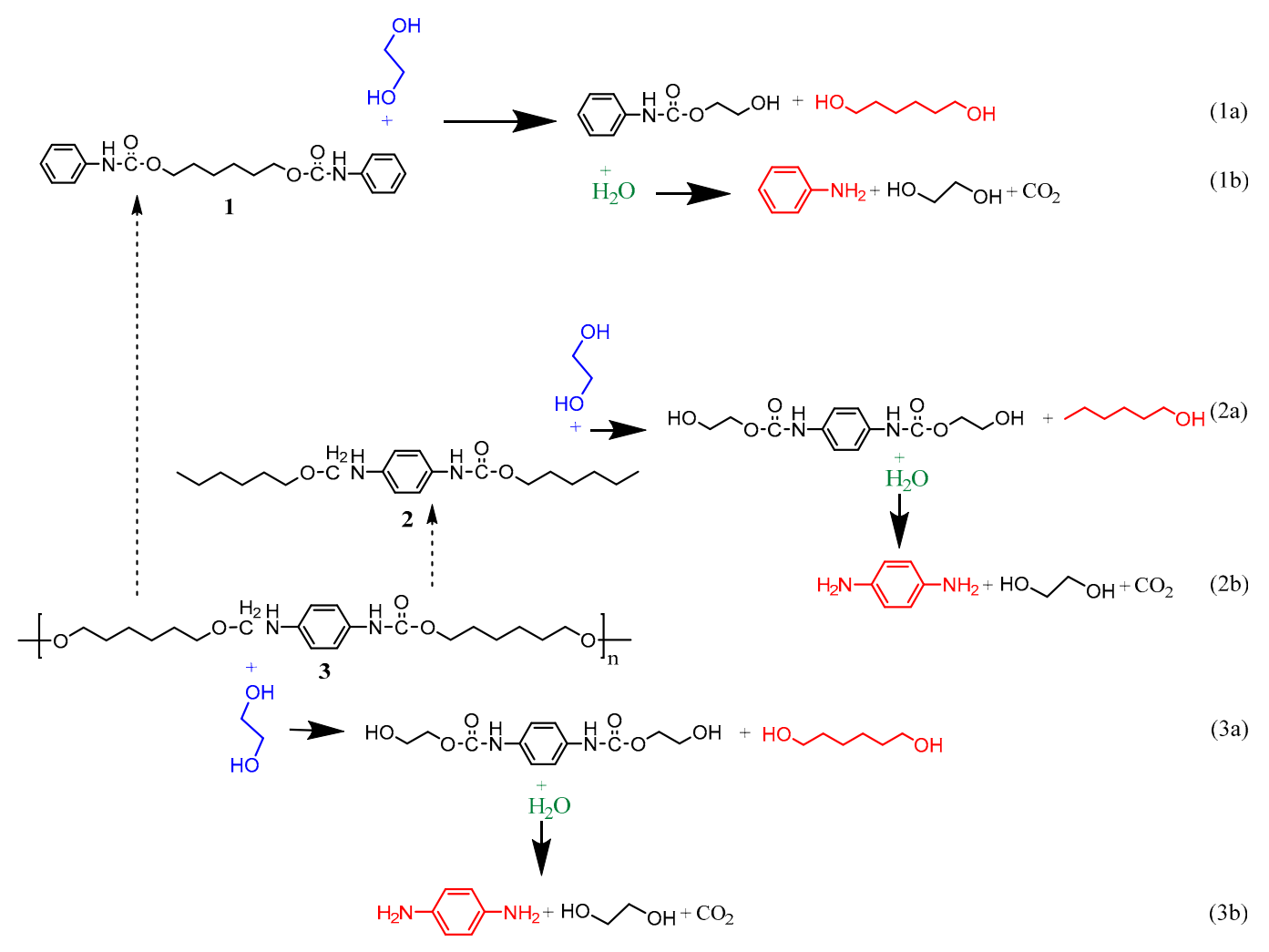

Scheme 5. Overview for multistage chemical recycling of model polyurethane and model dicarbamates implementing first glycolysis and then the hydrolysis reaction. Based on the reactions, both polyols and amines are recovered when the whole procedure is completed.

\subsection{Glycolysis}

The glycolysis reaction is carried out in a three-neck flask equipped with a magnetic stirrer and reflux condenser under argon atmosphere in an oil bath. Ethylene glycol was used as the glycolysis agent, dried using $3 \AA$ molecular sieves to avoid possible interference with the results by unwanted amine formation due to the presence of water. Five parts of ethylene glycol were used with respect to the mass of polyurethane or dicarbamate and reacted at the desired temperature with $10 \mathrm{wt}$. \% of sodium acetate as the catalyst for two hours. After two hours, to separate the newly formed carbamates and remaining oligomers, the mixture was washed with water and filtered, as these components are insoluble in water. The precipitate on the filter was dried and then used for the next step of hydrolysis.

\subsection{Hydrolysis}

The hydrolysis reaction was done in an Anton Paar Synthos 3000 microwave reactor. For this reaction, $10 \mathrm{~mL}$ of water, adjusted to $\mathrm{pH} 11$ using sodium hydroxide as the catalyst, and the precipitate from the previous glycolysis were added to $80 \mathrm{~mL}$ microwave vials. Then, the temperature was set to $200{ }^{\circ} \mathrm{C}$, and additionally for safety reasons, the maximum power was chosen at 800 watt and maximum pressure at 40 bar. The reaction was carried out for $90 \mathrm{~min}$ under air. When the reaction was completed, the amines and compounds formed were then extracted from the water phase using methyl-tert-butyl-ether (MTBE) and the solvent was evaporated using a rotary evaporator.

\subsection{Characterization Techniques}

To assess the unreacted isocyanates that remain in the polyurethane or dicarbamates during the synthesis, Fourier-transform infrared spectroscopy (FT-IR) was used. For this measurement, small solid samples of each component were placed on the ATR crystal of an FT-IR Bruker Lumos, and 32 scans were performed for each sample. 
Gas chromatography-mass spectrometry (GC-MS) was used for the determination of different, more-volatile components as well as their quantity in the mixture after the glycolysis and hydrolysis. It should be noted that phenylene diamine obtained after hydrolysis could not be detected with GC-FID and so this method of characterization was not used for its quantification. For characterization with GC-MS, samples from mixtures were dissolved in acetone at a concentration of $1 \mathrm{mg} \cdot \mathrm{mL}^{-1}$, filtered with a pore size of $0.45 \mu \mathrm{m}$ and injected to the GC column at an injector temperature of $320{ }^{\circ} \mathrm{C}$. The oven temperature program starts from $50{ }^{\circ} \mathrm{C}$ and reaches $320^{\circ} \mathrm{C}$ at a rate of $10^{\circ} \mathrm{C} / \mathrm{min}$ and was held at $320^{\circ} \mathrm{C}$ for $20 \mathrm{~min}$. The characterization was carried out on a Shimadzu GCMSQP2010S equipped with a Zebron ZB-5MS (30 $\mathrm{m} \times 0.25 \mathrm{~mm}$ i.d., $0.25 \mu \mathrm{m}$ film thickness) capillary column (from Phenomenex, The Netherlands).

Gel permeation chromatography (GPC) was used for the determination of the approximate size of the different components in the glycolysis mixture. For this characterization, aliquots from the glycolysis mixture were dissolved in tetrahydrofuran (THF) at a concentration of $5 \mathrm{mg} \cdot \mathrm{mL}^{-1}$ and then filtered with a pore size of $0.45 \mu \mathrm{m}$. The analysis was carried out on an Agilent 1100 series system equipped with a $100 \AA$ Phenogel column (300 $\times 7.80 \mathrm{~mm}, 5$ micron) (Phenomenex, Utrecht, The Netherlands) connected to a refractive index detector.

\section{Results and Discussion}

\subsection{Polyurethane and Dicarbamates}

The synthesized polyurethane as well as both dicarbamates were characterized via FT-IR in order to investigate the urethane bond formation and also the possibility of remaining isocyanates in the products. The spectra of (1), (2), and (3) are available in the Supplementary Materials (Figure S1). It can be seen from all three spectra that there is no peak around $2200 \mathrm{~cm}^{-1}$, which indicates there is no free isocyanate left in the products. On the other hand, the peaks in the range of $3500-3200 \mathrm{~cm}^{-1}$ are related to $\mathrm{N}-\mathrm{H}$ stretching, the peaks in the range of $1730-1640 \mathrm{~cm}^{-1}$ are related to $\mathrm{C}=\mathrm{O}$ vibrations and the peaks around 2930 and $2860 \mathrm{~cm}^{-1}$ are related to the symmetric and asymmetric vibrations of $\mathrm{C}-\mathrm{H}$ bonds, respectively. It should also be noted that the peaks present below $1600 \mathrm{~cm}^{-1}$ are characteristic for the presence of the urethane bond: around $1514 \mathrm{~cm}^{-1} \mathrm{C}-\mathrm{N}$ and $\mathrm{N}-\mathrm{H}$, around $1214 \mathrm{~cm}^{-1} \mathrm{C}-\mathrm{N}$, and around $1068 \mathrm{~cm}^{-1} \mathrm{C}-\mathrm{O}-\mathrm{C}=\mathrm{O}[46,47]$.

\subsection{First Step-Polyol Recovery}

Repeating the glycolysis reaction (the reaction pathways are presented in Scheme 5, marked as reactions (1a), (2a), and (3a) for compounds (1), (2), and (3), respectively) and assessing the results for optimization of the temperature, the ideal temperature for the glycolysis of polyurethane turned out to be $190{ }^{\circ} \mathrm{C}$, and for the dicarbamates, $170{ }^{\circ} \mathrm{C}$. In order to study the glycolysis process, samples from the glycolysis mixture for polyurethane and dicarbamates were taken after two hours of reaction and were analyzed by means of GPC and GC-MS.

The alcohol and diol are recovered after two hours of glycolysis of each compound, which means that the first desired product of the chemical recycling is obtained from the glycolysis step. On the other hand, there are some peaks other than the polyol or ethylene glycol present in the GPC chromatograms, which are related to the newly formed carbamates and oligomers during the glycolysis. These products are the desired raw materials for the future step of hydrolysis. The GPC chromatograms are available in the Supplementary Materials marked as Figures S2-S4 for the glycolysis mixture of dicarbamates (1 and 2) polyurethane (3), respectively, overlapped with chromatograms of 1,6-hexanediol and 1-hexanol. The peak around a retention time of $18.2 \mathrm{~min}$ is related to the excess ethylene glycol in the mixture, while retention times around 16.6 and $17.1 \mathrm{~min}$ are linked to 1,6-hexanediol and 1-hexanol, respectively.

Figure 1 shows the GC-MS chromatograms for the glycolysis of polyurethane (3) (Figure 1a), dicarbamate (1) (Figure 1b), and dicarbamate (2) (Figure 1c), respectively. 
Recovered polyols are visible in the chromatograms, which is consistent with the data from GPC. On the other hand, hexanoic acid has been created during the glycolysis as a byproduct of the reaction. Furthermore, during the glycolysis of both dicarbamates, amines were formed. By the use of molecular sieves, the absence of water is ensured during the reaction, so it can be concluded that the amines formed were due to the thermal degradation of carbamates during the glycolysis, which is also mentioned in available literature [24,48]. It should be noted that larger oligomers and larger carbamates are not detectable in the GC as they are insufficiently volatile-and so only in the result from glycolysis of dicarbamate (1) the newly formed ethyl carbamate and ethyl dicarbamate are available. Furthermore, at high injector temperature, the carbamates decompose, which is the reason why phenyl isocyanate is also visible in the chromatogram - in accordance with literature data $[49,50]$.
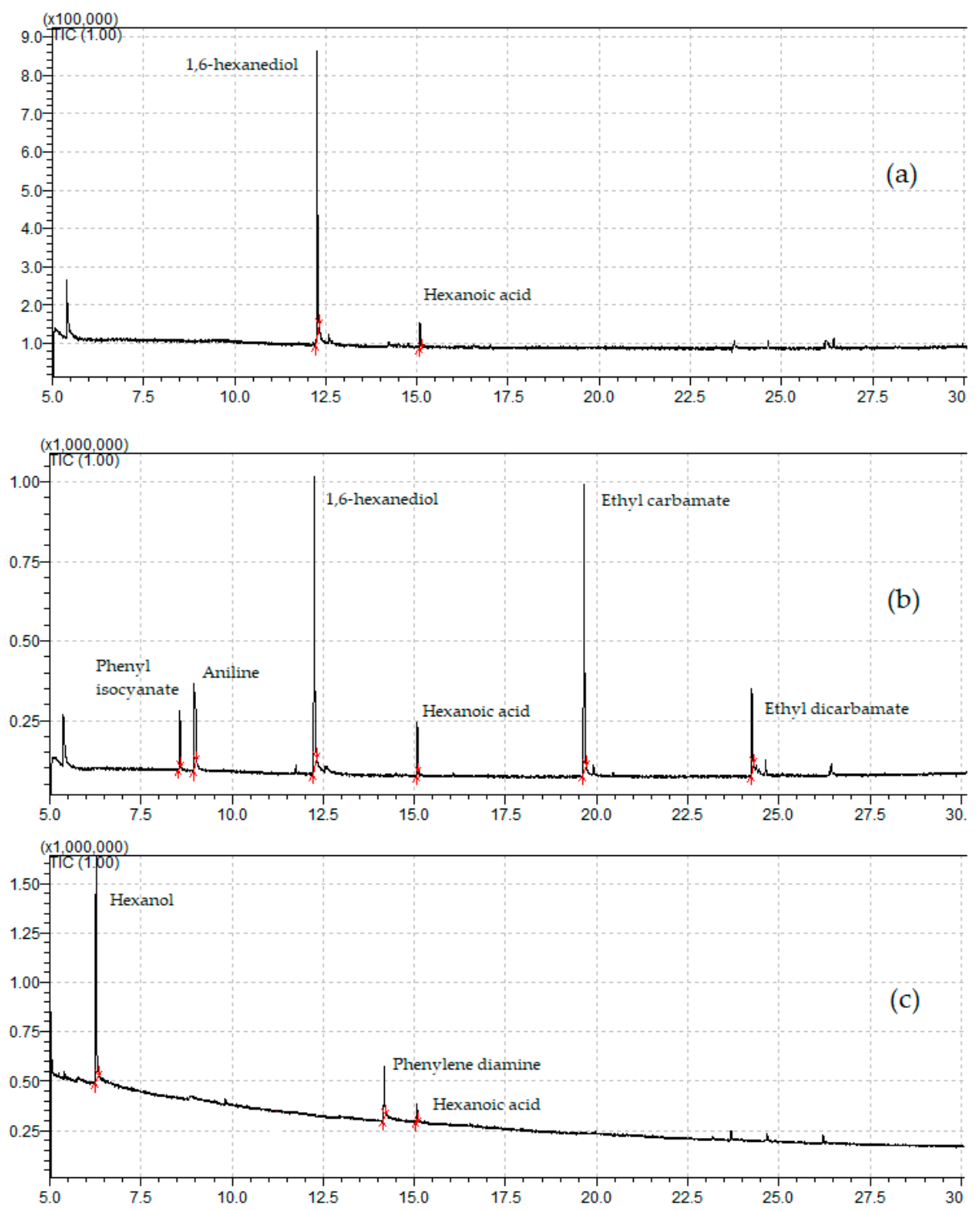

Figure 1. GC-MS result of glycolysis mixture for recycling of polyurethane (3) (chromatogram (a)), dicarbamate (1) (chromatogram (b)), and dicarbamate (2) (chromatogram (c)).

\subsection{Second Step-Amine Recovery}

During the glycolysis reaction, smaller-sized carbamates and oligomers are generated, which can then be used in a hydrolysis process to generate amines. In this way, the initial 
isocyanates can be recovered as amines and reused for new products. In this study, as discussed previously, the glycolysis mixture was washed with water and filtered to extract the solid residue of glycolysis containing the newly formed carbamates and urethane oligomers. Then, this solid was used for the hydrolysis in the microwave reactor at high pressure and temperature. Figure 2 depicts the GC chromatograms of the extracted hydrolysis product for polyurethane (3) (Figure 2a), dicarbamate (1) (Figure 2b), and dicarbamate (2) (Figure 2c). The reaction pathways are also present on Scheme 5 marked as reactions (1b), (2b), and (3b) for compounds (1), (2), and (3) respectively. It is shown that the products of the hydrolysis were both the amine and polyol or alcohol, which is related to the remaining oligomers after glycolysis. Ethylene glycol in not visible on the chromatograms since it was eluted together with solvent at the beginning.
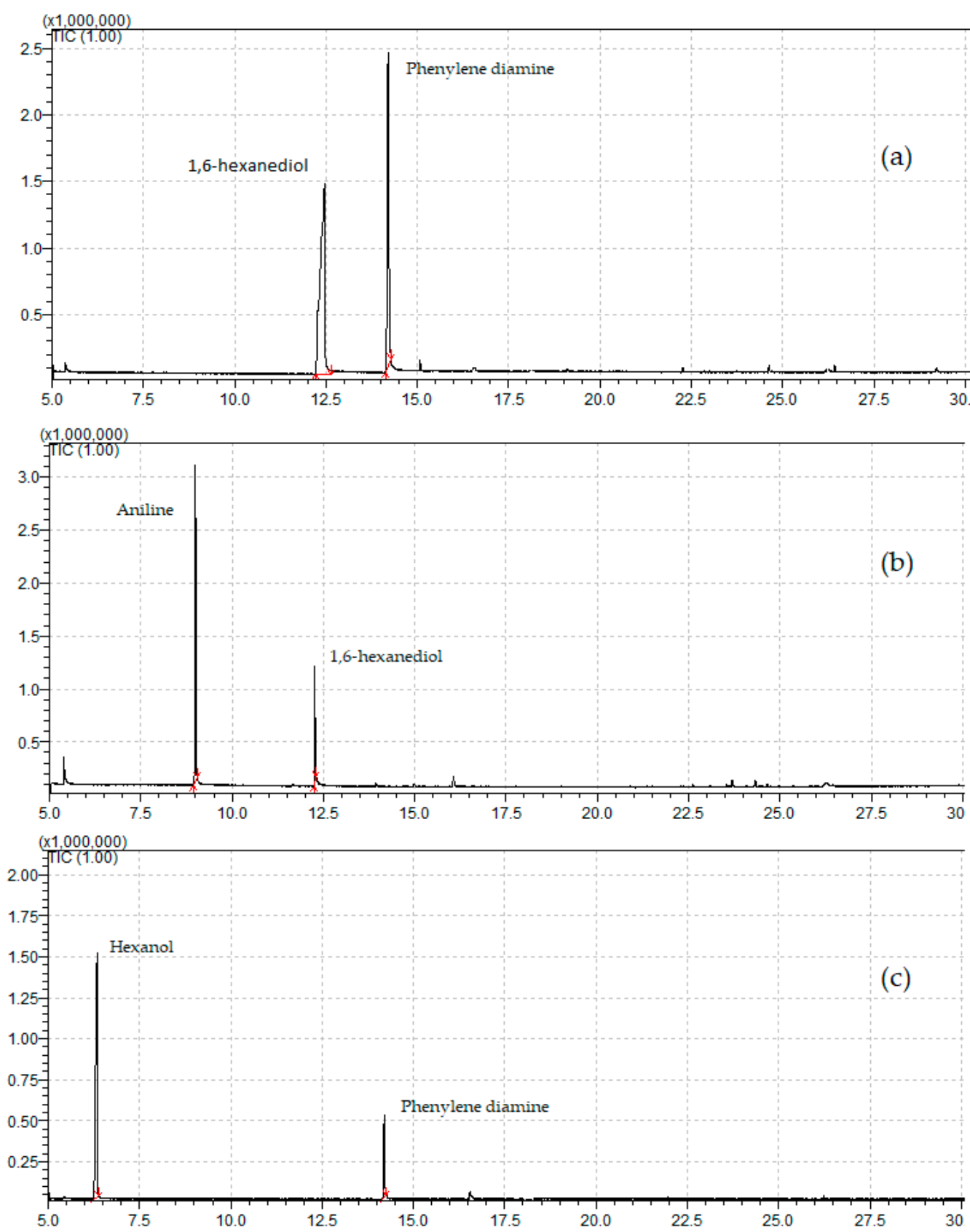

Figure 2. GC-MS result of the hydrolysis product for recycling of polyurethane (3) (a), dicarbamate (1) (b), and dicarbamate (2) (c). 
In order to obtain quantitative information, an internal standard was used, and the relative response factors (RRF) for the amine and polyol or alcohol were calculated, due to the weak response factor of amine in comparison to that for the polyol or alcohol [51]. In the first step, the relative response factors for aniline, phenylene diamine, 1-hexanol, and 1,6-hexanediol were calculated. As internal standard, ethyl hexanoate (+99\%, analytical standard) was used. In the second step, the internal standard was added to the hydrolysis products and analyzed on GC-FID to calculate the amount of aniline and 1,6hexanediol after the glycolysis-hydrolysis process of hexane-1,6-diyl bis(phenylcarbamate) (1), phenylene diamine, and 1-hexanol after the glycolysis-hydrolysis process of dihexyl 1,4-phenylenedicarbamate (2) and, finally, phenylene diamine and 1,6-hexanediol after the glycolysis-hydrolysis process of model polyurethane (3). The yield of the amine was then calculated as the molar amount of the produced amine with respect to the initial number of moles of isocyanate in the starting material. Results are given in Table 1. Although the yield of amine production during the second step of hydrolysis was not quantitative, at least part of the original isocyanate components could be recovered, and this amount may be further improved by optimization of the reaction pressure and temperature, or by using a more effective catalyst. It should also be noted that, during the extraction procedure for obtaining the solid fraction from the glycolysis, part of this solid is lost, which is also a reason for the low yield.

Table 1. Glycolysis-hydrolysis reaction yields.

\begin{tabular}{cccccc}
\hline Substrate & $\begin{array}{c}\text { Mass of } \\
\text { Substrate (g) }\end{array}$ & $\begin{array}{c}\text { Initial Amount of } \\
\text { Isocyanate (mmol) }\end{array}$ & $\begin{array}{c}\text { Mass of } \\
\text { Product (g) }\end{array}$ & $\begin{array}{c}\text { Amount of Produce } \\
\text { Amine (mmol) }\end{array}$ & Yield (\%) $^{\mathbf{2}}$ \\
\hline Polyurethane (3) & 3.65 & 13.10 & 0.54 & 3.80 & 29 \\
Dicarbamate (2) & 5.40 & 14.80 & 0.59 & 4.50 & 33 \\
Dicarbamate (1) & 4.80 & 27.00 & 1.18 & 11.10 & 41 \\
\hline
\end{tabular}

${ }^{1}$ Determined after extraction, ${ }^{2}$ Determined by GC-FID analysis.

The multistage recycling of polyurethane would enable us to recover polyols and amines separately. In this way, the obtained chemicals are less contaminated, and we can use the advantages of split-phase glycolysis, which is not possible in the case of hydroglycolysis on polyurethane. In addition, as the carbamates obtained from the glycolysis process are smaller in size in comparison to the original polyurethane, the hydrolysis process for them can be done under milder conditions than the conventional hydrolysis, which makes this process interesting for future applications. The importance of further research in this area is supported by several recent publications about PU recycling [52-54], moreover projects are at a high technology readiness level on a European scale nowadays [55].

\section{Conclusions}

By means of the prevalent chemical recycling methods for polyurethane, only polyols are recovered and a large and valuable part of the original feedstock (the isocyanate part) is lost. In order to recover this part as well, multistage chemical recycling of polyurethane is proposed here, conducting first glycolysis and then hydrolysis on the carbamate fraction. To avoid the complications caused by the chemical complexity of industrial polyurethanes, a simple, model polyurethane and its relevant dicarbamate oligomers were synthesized. The glycolysis on these compounds was effected with ethylene glycol. The newly formed carbamates and carbamate oligomers after glycolysis were precipitated and subsequently hydrolyzed at $200{ }^{\circ} \mathrm{C}$ in order to regain the original isocyanate fraction as their amines. The overall amine yields after glycolysis-hydrolysis range around $30-40 \%$ in the non-optimized initial attempt. These yields will likely be further improved in future research by further optimization of the reaction parameters. 
Supplementary Materials: The following are available online at https:/ / www.mdpi.com/2071-1 050/13/6/3583/s1, Figure S1: FT-IR results of model polyurethane (3) and dicarbamates (1 and 2);Figure S2: GPC chromatogram of the glycolysis result for dicarbamate (1) (blue peaks) overlap with chromatogram of 1,6-hexanediol (red peak); Figure S3: GPC chromatogram of the glycolysis result for carbamate (2) (blue peaks) overlap with chromatogram of 1-hexanol (red peak); Figure S4: GPC chromatogram of the glycolysis result for polyurethane (3) (blue peaks) overlap with chromatogram of 1,6-hexanediol (red peak).

Author Contributions: Conceptualization, L.P., C.M.L.V.V., and P.B.; methodology, P.Z., L.P., C.M.L.V.V., and P.B.; validation, L.P.; formal analysis, P.Z. and L.P.; investigation, P.Z.; resources, L.P.; writingoriginal draft preparation, P.Z. and L.P.; writing—review and editing, C.M.L.V.V. and P.B.; supervision, C.M.L.V.V. and P.B.; funding acquisition, L.P., C.M.L.V.V., and P.B. All authors have read and agreed to the published version of the manuscript.

Funding: This research was funded by the University of Antwerp, BOF-STIMPRO: "CycloPUR", Project ID: 39861.

Institutional Review Board Statement: Not applicable.

Informed Consent Statement: Not applicable.

Data Availability Statement: Data are contained within the article or Supplementary Materials.

Acknowledgments: The authors gratefully acknowledge the University of Antwerp for financial support of this project.

Conflicts of Interest: The authors declare no conflict of interest. The funders had no role in the design of the study; in the collection, analyses, or interpretation of data; in the writing of the manuscript; or in the decision to publish the results.

\section{References}

1. Austin, A.; Hicks, D.A. A review of the global PU industry 2016 and outlook for 2017. PU Mag. 2017, 14, 1-15.

2. Plastics Europe European Plastics Industry Market Data. Available online: https://www.plasticseurope.org/en/resources/ market-data (accessed on 21 February 2021).

3. Sharmin, E.; Zafar, F. Polyurethane: An Introduction. In Polyurethane; Intech: Rijeka, Croatia, 2012; Volume 80, pp. 76-78. [CrossRef]

4. Bayer, O. Das Di-Isocyanat-Polyadditionsverfahren (Polyurethane). Angew. Chem. 1947, 59, 257-272. [CrossRef]

5. Akindoyo, J.O.; Beg, M.D.H.; Ghazali, S.; Islam, M.R.; Jeyaratnam, N.; Yuvaraj, A.R. Polyurethane types, synthesis and applications-A review. RSC Adv. 2016, 6, 114453-114482. [CrossRef]

6. Behrendt, G.; Naber, B.W. The chemical recycling of polyurethanes. J. Univ. Chem. Technol. Metall. 2009, 44, 3-23.

7. Anuar Sharuddin, S.D.; Abnisa, F.; Wan Daud, W.M.A.; Aroua, M.K. A review on pyrolysis of plastic wastes. Energy Convers. Manag. 2016, 115, 308-326. [CrossRef]

8. Brems, A.; Baeyens, J.; Dewil, R. Recycling and recovery of post-cetalonsumer plastic solid waste in a European context. Therm. Sci. 2012, 16, 669-685. [CrossRef]

9. DeGaspari, J. From Trash to Cash. Mech. Eng. 1999, 121, 48-51. [CrossRef]

10. Datta, J.; Włoch, M. Chapter 14-Recycling of Polyurethanes. In Polyurethanes Polymers; Sabu, T., Datta, J., Haponiuk, J.T., Reghunadhan, A., Eds.; Elsevier: Amsterdam, The Netherlands, 2017; pp. 323-358. [CrossRef]

11. Gradus, R.H.J.M.; Nillesen, P.H.L.; Dijkgraaf, E.; van Koppen, R.J. A Cost-effectiveness Analysis for Incineration or Recycling of Dutch Household Plastic Waste. Ecol. Econ. 2017, 135, 22-28. [CrossRef]

12. Shen, L.; Worrell, E. Chapter 13-Plastic Recycling. In Handbook of Recycling; Worrell, E., Reuter, M.A., Eds.; Elsevier: Amsterdam, The Netherlands, 2014; pp. 179-190. [CrossRef]

13. Zia, K.M.; Bhatti, H.N.; Ahmad Bhatti, I. Methods for polyurethane and polyurethane composites, recycling and recovery: A review. React. Funct. Polym. 2007, 67, 675-692. [CrossRef]

14. Zevenhoven, R. Treatment and Disposal of Polyurethane Wastes: Options for Recovery and Recycling; Techical Report; Helsinki University of Technology Department of Mechanical Engineering: Helsinki, Finland, 2004.

15. Nikje, M.M.A.; Garmarudi, A.B.; Idris, A.B. Polyurethane waste reduction and recycling: From bench to pilot scales. Des. Monomers Polym. 2011, 14, 395-421. [CrossRef]

16. Kinoshita, O. Process for Decomposition of a Polyurethane Resin. U.S. Patent US3632530A, 4 January 1972.

17. Broeck, T.R.T.; Peabody, D.W. Method for Reclaiming Cured Cellular Polyurethanes. U.S. Patent US2937151A, 17 May 1960.

18. Bărbălată, A.; Bărbíntă, V.; Caraculacu, A.A. On the mechanism of isocyanate preparation by the phosgenation reaction. React. Kinet. Catal. Lett. 1981, 18, 427-430. [CrossRef]

19. Salloum, R.J.; Duff, C.C. Method for Reclaiming Polyurethane Foam. U.S. Patent US4328368A, 4 May 1982. 
20. Dai, Z.; Hatano, B.; Kadokawa, J.I.; Tagaya, H. Effect of diaminotoluene on the decomposition of polyurethane foam waste in superheated water. Polym. Degrad. Stab. 2002, 76, 179-184. [CrossRef]

21. Motokucho, S.; Nakayama, Y.; Morikawa, H.; Nakatani, H. Environment-friendly chemical recycling of aliphatic polyurethanes by hydrolysis in a CO2-water system. J. Appl. Polym. Sci. 2018, 135, 14-17. [CrossRef]

22. Simón, D.; Borreguero, A.M.; de Lucas, A.; Rodríguez, J.F. Recycling of polyurethanes from laboratory to industry, a journey towards the sustainability. Waste Manag. 2018, 76, 147-171. [CrossRef]

23. Marković, V.; Hicks, D.A. Design for chemical recycling. Philos. Trans. R. Soc. A Math. Phys. Eng. Sci. 1997, 355, 1415-1424. [CrossRef]

24. Borda, J.; Pásztor, G.; Zsuga, M. Glycolysis of polyurethane foams and elastomers. Polym. Degrad. Stab. 2000, 68, 419-422. [CrossRef]

25. Zhu, P.; Cao, Z.B.; Chen, Y. Glycolysis recycling of rigid waste polyurethane foam from refrigerators. Environ. Technol. 2014, 35, 2676-2684. [CrossRef]

26. Alavi Nikje, M.M.; Nikrah, M.; Haghshenas, M. Microwave assisted “split-phase" glycolysis of polyurethane flexible foam wastes. Polym. Bull. 2007, 59, 91-104. [CrossRef]

27. Molero, C.; De Lucas, A.; Rodríguez, J.F. Recovery of polyols from flexible polyurethane foam by "split- phase" glycolysis with new catalysts. Polym. Degrad. Stab. 2006, 91, 894-901. [CrossRef]

28. Molero, C.; de Lucas, A.; Rodríguez, J.F. Recovery of polyols from flexible polyurethane foam by "split-phase" glycolysis: Study on the influence of reaction parameters. Polym. Degrad. Stab. 2008, 93, 353-361. [CrossRef]

29. Simón, D.; García, M.T.; De Lucas, A.; Borreguero, A.M.; Rodríguez, J.F. Glycolysis of flexible polyurethane wastes using stannous octoate as the catalyst: Study on the influence of reaction parameters. Polym. Degrad. Stab. 2013, 98, 144-149. [CrossRef]

30. Modesti, M.; Costantini, F.; dal Lago, E.; Piovesan, F.; Roso, M.; Boaretti, C.; Lorenzetti, A. Valuable secondary raw material by chemical recycling of polyisocyanurate foams. Polym. Degrad. Stab. 2018, 156, 151-160. [CrossRef]

31. Pang, Y.; Liu, X.; Li, Y.; Cui, T.; Liu, X.; Li, Q.; Wan, H.; Mao, A. Solvent Decomposition of Polyurethane Foam Obtained from Waste Upholstered Furniture to Recover Polyols. Am. J. Polym. Sci. Technol. 2019, 5, 88-96. [CrossRef]

32. Wu, C.H.; Chang, C.Y.; Li, J.K. Glycolysis of rigid polyurethane from waste refrigerators. Polym. Degrad. Stab. 2002, 75, 413-421. [CrossRef]

33. Johnston, A.R. The Toxic Effects of Amines. J. Infect. Dis. 1928, 42, 473-484. [CrossRef]

34. Eide-Haugmo, I.; Brakstad, O.G.; Hoff, K.A.; Sørheim, K.R.; da Silva, E.F.; Svendsen, H.F. Environmental impact of amines. Energy Procedia 2009, 1, 1297-1304. [CrossRef]

35. Tashkinova, I.; Batrakova, G. Biodegradation of Toxic Aromatic Amines in Industrial Site Soils. In Proceedings of the 4th International Conference on Environmental Pollution and Remediation, Prague, Czech Republic, 11-13 August 2014. Paper No. 98.

36. Simón, D.; Borreguero, A.M.; de Lucas, A.; Molero, C.; Rodríguez, J.F. Novel polyol initiator from polyurethane recycling residue. J. Mater. Cycles Waste Manag. 2013, 16, 525-532. [CrossRef]

37. Faust, S.D.; Gomaa, H.M. Chemical hydrolysis of some organic phosphorus and carbamate pesticides in aquatic environments. Environ. Lett. 1972, 3, 171-201. [CrossRef]

38. Attig, J.B.; Ouertani, R.; Megriche, A.; Hamida, N.B.; El Atrache, L.L. Kinetic Study and Mechanism Hydrolysis of 4-Bromo-3,5 dimethylphenyl N-methylcarbamate in Aqueous Media. Int. J. Chem. Kinet. 2017, 49, 761-769. [CrossRef]

39. Lin, X.; Hong, Q.; Wu, X.; Guo, L.; Xie, Z. Analysis of phenoxyl-type N-methylcarbamate pesticide residues in vegetables by capillary zone electrophoresis with pre-column hydrolysis and amperometric detection. J. Chromatogr. Sci. 2008, 46, 615-621. [CrossRef]

40. Cao, V.; Schaffer, M.; Licha, T. The feasibility of using carbamates to track the thermal state in geothermal reservoirs. Geothermics 2018, 72, 301-306. [CrossRef]

41. Aou, K.; Schrock, A.K.; Ginzburg, V.V.; Price, P.C. Characterization of polyurethane hard segment length distribution using soft hydrolysis/MALDI and Monte Carlo simulation. Polymer 2013, 54, 5005-5015. [CrossRef]

42. Gerlock, J.L.; Braslaw, J.; Albright, J. Catalyzed Dissolution-Hydrolysis of Polyurethane Wastes. U.S. Patent US4317939A, 2 March 1982.

43. Gerlock, J.; Braslaw, J.; Zinbo, M. Polyurethane Waste Recycling. 1. Glycolysis and Hydroglycolysis of Water-Blown Foams. Ind. Eng. Chem. Process. Des. Dev. 1984, 23, 545-552. [CrossRef]

44. Alavi Nikje, M.M.; Nikrah, M.; Mohammadi, F.H.A. Microwave-assisted polyurethane bond cleavage via hydroglycolysis process at atmospheric pressure. J. Cell. Plast. 2008, 44, 367-380. [CrossRef]

45. Lyman, D.J.; Polyurethanes, I. The solution polymerization of diisocyanates with ethylene glycol. J. Polym. Sci. 1960, 45, 49-59. [CrossRef]

46. Dhaliwal, G.S.; Anandan, S.; Chandrashekhara, K.; Lees, J.; Nam, P. Development and characterization of polyurethane foams with substitution of polyether polyol with soy-based polyol. Eur. Polym. J. 2018, 107, 105-117. [CrossRef]

47. Rehman, F. Synthesis and Characterization of Speciality Polyurethane Elastomers. Ph.D. Thesis, University of Agriculture, Faisalabad, Pakistan, 2010.

48. Lee, S.H.; Ohkita, T.; Teramoto, Y. Polyol recovery from biomass-based polyurethane foam by glycolysis. J. Appl. Polym. Sci. 2005, 95, 975-980. [CrossRef] 
49. Wang, B.; He, J.; Sun, R.C. Carbamate synthesis from amines and dialkyl carbonate over inexpensive and clean acidic catalystSulfamic acid. Chin. Chem. Lett. 2010, 21, 794-797. [CrossRef]

50. Sakaue, S. Direct gas chromatographic determination of carbamate pesticides and their formulation. Agric. Biol. Chem. 1987, 51, 1239-1245. [CrossRef]

51. Jenke, D.; Odufu, A. Utilization of internal standard response factors to estimate the concentration of organic compounds leached from pharmaceutical packaging systems and application of such estimated concentrations to safety assessment. J. Chromatogr. Sci. 2012, 50, 206-212. [CrossRef]

52. Zhao, L.; Semetey, V. Recycling Polyurethanes through Transcarbamoylation. ACS Omega 2021, 6, 4175-4183. [CrossRef] [PubMed]

53. Vanbergen, T.; Verlent, I.; De Geeter, J.; Haelterman, B.; Claes, L.; De Vos, D. Recycling of flexible polyurethane foam by split-phase alcoholysis: Identification of additives and alcoholyzing agents to reach higher efficiencies. Chemsuschem 2020, 13, 3835-3843. [CrossRef] [PubMed]

54. Deng, Y.; Dewil, R.; Appels, L.; Ansart, R.; Baeyens, J.; Kang, Q. Reviewing the thermo-chemical recycling of waste polyurethane foam. J. Environ. Manag. 2021, 278, 111527. [CrossRef] [PubMed]

55. PUReSmart-Research project of Horizon 2020 Research and Innovation Program under Grant Agreement N ${ }^{\circ} 814543$. Available online: https: / www.puresmart.eu/ (accessed on 16 March 2021). 